\title{
Spinal cord compression revealing fluorosis
}

\section{Kherfani Abdelhakim, Amri Khalil*, Maatar Nidhal, Mestiri Mondher, Bouaziz Mouna, Ladeb Mohamed}

Orthopaedic Institute M T Kassab, Tunisia

\section{Email address:}

akhali1.kh@gmail.com (Amri. K)

\section{To cite this article:}

Kherfani Abdelhakim, Amri Khalil, Maatar Nidhal, Mestiri Mondher, Bouaziz Mouna, Ladeb Mohamed. Spinal Cord Compression Revealing Fluorosis. Journal of Surgery. Vol. 2, No. 1, 2014, pp. 4-6. doi: 10.11648/j.js.20140201.12

\begin{abstract}
Bone fluorosis due to high water fluoride contents is endemic in North Africa. Neurological complications as spinal cord compression at dorsal level are rare and unusual. Its outcome depends on early diagnosis and surgical treatment. The authors report a new case of spinal cord compression due to ossification of the yellow vertebral ligament in a patient with previously unknown bone fluorosis.
\end{abstract}

Keywords: Spinal Cord Compression, Fluorosis, Ossification of the Yellow Vertebral Ligament

\section{Introduction}

Bone fluorosis due to high water fluoride contents is endemic in North Africa [1]. Neurological complications as spinal cord compression are rare, predominating at the cervical spine. Spinal cord compression at the dorsal level is unusual [2], and its outcome depends on early diagnosis and surgical treatment.

The authors report a new case of spinal cord compression due to ossification of the yellow vertebral ligament in a patient with previously unknown bone fluorosis.

\section{Case-Report}

A 50 years old man, from southern Tunisia with unremarkable medical history, presented after a dorsal spine moderate trauma with a monoplegia of the right lower limb and urinary incontinence. Physical examination showed brownish discoloration of the teeth and marked pyramidal signs in the right lower limb.

Spine radiographs disclosed a moderate diffuse vertebral sclerosis (Fig 1). Magnetic resonance imaging (MRI) was performed in the emergency room to assess neurological impairment. It disclosed compression of the dorsal spine cord at T8, T9 and T10 level with a low T1 and T2 process originating from the posterior arch of the vertebra. Computed tomography was performed to assess this posterior spinal abnormality and showed ossification of the yellow ligament (Fig 2) and (Fig 3).
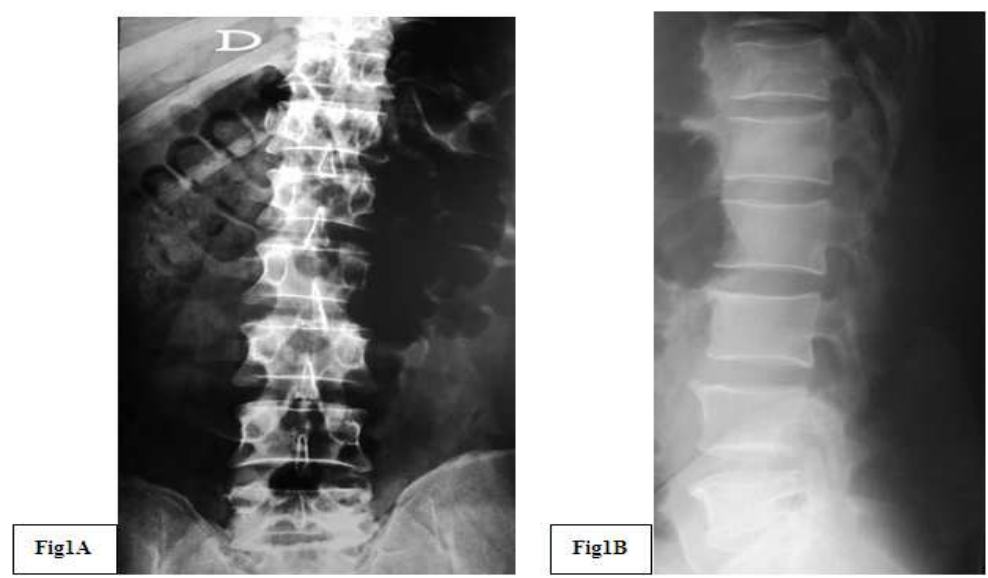

Figure 1. Lumbar spine radiographs. Anteroposterior (a) and lateral projection (b): Moderate diffuse bone sclerosis. 
Fluoride poisoning was suspected based on the geographic origin of the patient, brownish discoloration of the teeth, and imaging findings.

Fluoride assays showed marked elevation in urine (10, $44 \mathrm{mg} / \mathrm{g}$ creatinine, normal, $<0.5 \mathrm{mg} / \mathrm{g}$ creatinine).
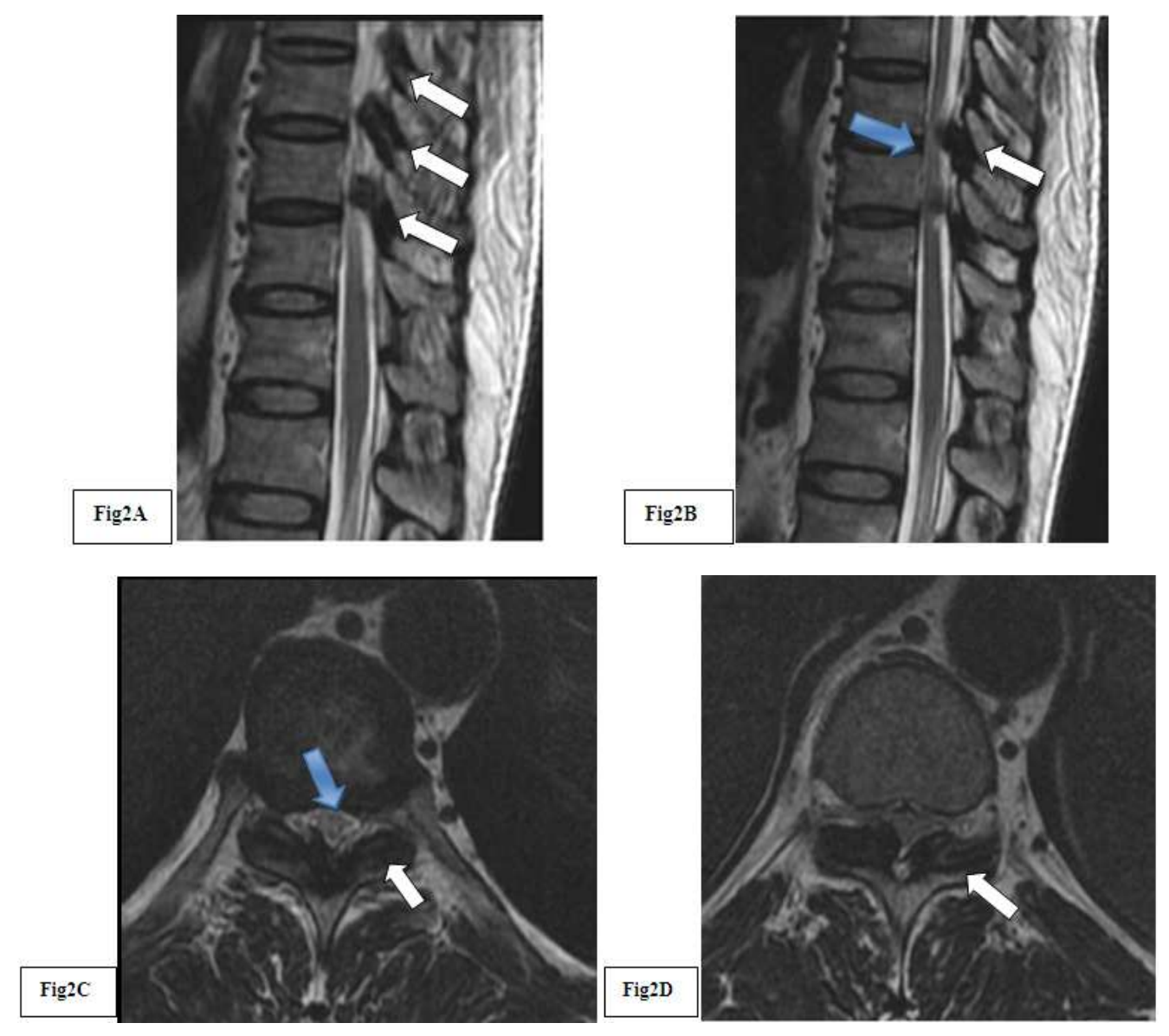

Figure 2. MRI of thoracic spine in T2-weighted sequences: sagittal section (a, b) axial sections through T9 (c) and T10 (d): Hypertrophy and low signal intensity of yellow ligaments in T8, T9 and T10 level (white arrow) inducing spinal cord compression with high T2 signal of the spinal cord (blue arrow).
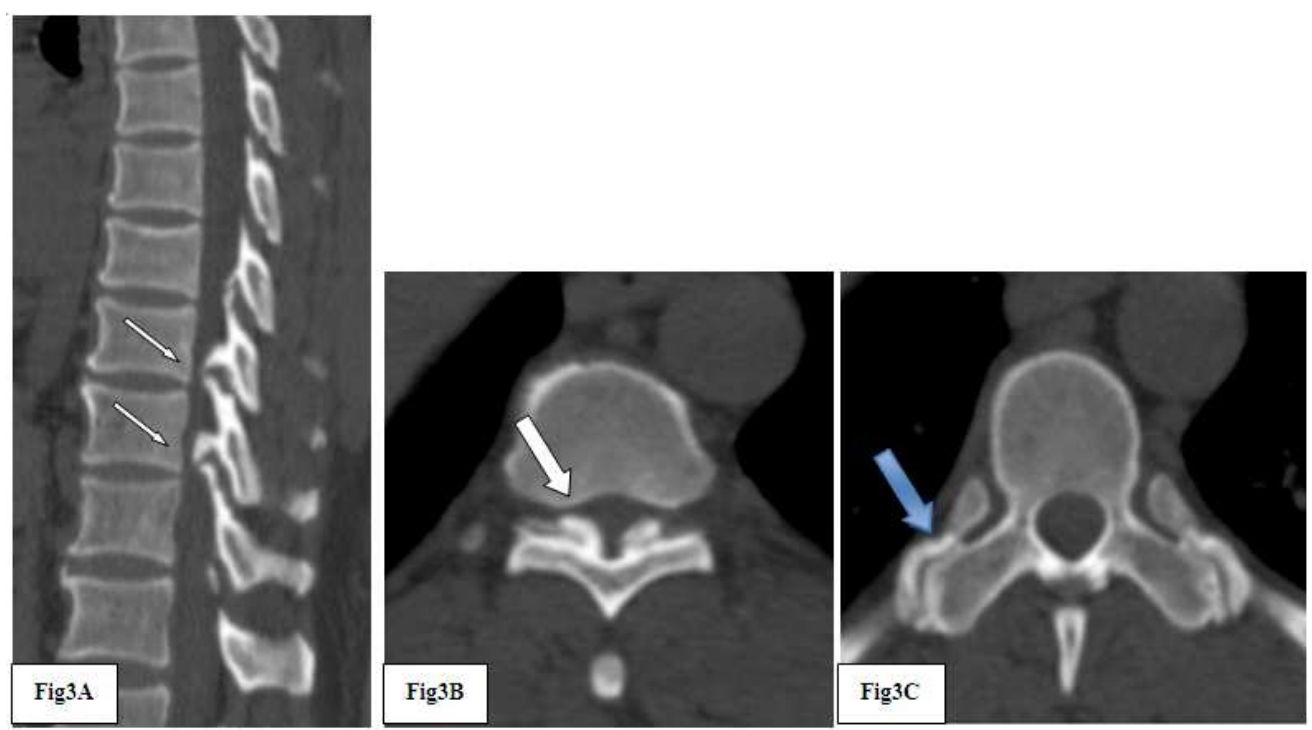

Figure 3. sagittal (a) and transversal (b, c) CT views of thoracic spine in bone algorithm: Calcification of yellow ligament in T8, T9 and T10 level (white arrow).Bilateral costotranversal T8 level arthropathy (blue arrow).

Surgical treatment was indicated, and the patient had multilevel decompression laminectomy at the dorsal spine presented significant neurological impairments recovery.

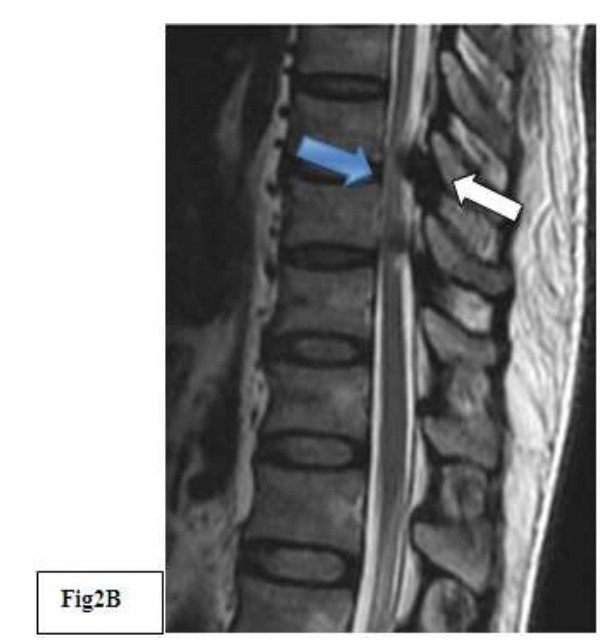




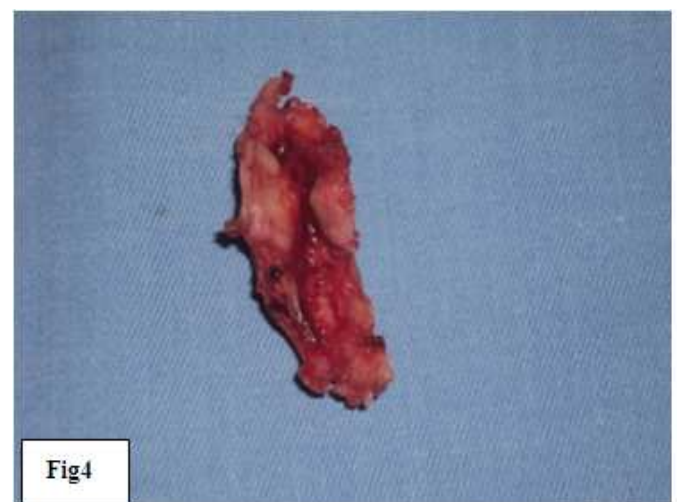

Figure 4. Photography of Surgery specimen showing calcification of the yellow ligament.

\section{Discussion}

Bone fluorosis due to high water fluoride contents is endemic in North Africa, such as southern and central Tunisia [1]. The precise incidence of bone fluorosis is unknown. Clinical symptoms are usually late and difficult to assess, bony pain predominating in lower limbs and cervical spine associated to a brownish teeth discoloration are a commons presenting symptoms, neurological impairment caused by spinal cord compression occurs in about $10 \%$ in patient with bony fluorosis after prolonged exposure to very high fluoride doses [2]. The diagnosis of bone fluorosis relies on imaging and biological features: diffuse bone sclerosis; ossification of interosseous membranes; high levels of fluoride in serum $(>0.2 \mathrm{mg} / \mathrm{L})$ and/or urine ( $1.8 \mathrm{mg} / 24 \mathrm{~h})$ [1-3].

In our case, the patient had no physical signs before the trauma; clinical presentation of fluorosis was unusual with acute and severe neurological impairment caused by spinal cord compression following a moderate trauma [4]. Thoracic spinal cord compression is very uncommon in fluorosis as neurological complications usually involve the cervical level.

Bone sclerosis and ossifications of ligaments and interosseous membranes are the main imaging signs. In patients with fluorosis, fluoride replaces the hydroxide ion within the hydroxyapatite crystal. In addition, fluoride stimulates osteoblast activity, markedly increasing the formation of bone, causing microcracksis. Subperiosteal bone formation, a hallmark of the disease, causes bone thickening and may narrow the spinal canal and foramina. Ossification may occur at sites of tendon and ligament attachment, most notably at the spine.

Neurological impairments complicating chronic fluorosis, best visualized by MRI [5], are often irreversible; but a favorable outcome may be achieved with early surgical treatment, as it has been showed in our case.

\section{Conclusion}

The outcome of patients with spinal cord compression due to fluorosis depends on the extent of the lesions and duration of poisoning. Diagnostic delay and prolonged fluoride exposure are associated with poor outcomes [6]. CT and MRI of the spine allow an early diagnosis and an accurate assessment of neurological complications. Early Surgery is mandatory in patients with spinal cord compression.

\section{References}

[1] Ghariani MT, Ben Hassen T, Djaidane A. La fluorose osseuse : à propos de 15 cas. Tunis Med 1983;4:292-5.

[2] Subba Rao B, Taraknath VR, Sista VN. Ossification of the posterior longitudinal ligament and fluorosis. J Bone Joint Surg 1992;74:469-70.

[3] Klisnick A, Clavelou P, Lemaire JJ, et al. Compression médullaire cervicale par ossification du ligament vertébral commun postérieur : à propos d'un cas. Neurochirurgie $1995 ; 41: 359-62$.

[4] Javier RM, Sibilia J, Durckel J, et al. Ostéopathies médicamenteuses. J Radiol 1999;80:709-13.

[5] Muthukumar N. Ossification of the ligamentum flavum as a result of fluorosis causing myelopathy: report of two cases. Neurosurgery 2005;56: E622.

[6] Jayakumar PN, Sastry Kolluri VR, Jam VK, et al. Ossified posterior longitudinal ligament. Neurol India 1987;35:55. 